\title{
Platelet-rich plasma shows beneficial effects for patients with knee osteoarthritis by suppressing inflammatory factors
}

\author{
GUILIN HUANG ${ }^{1}$, SHA HUA $^{2}$, TUANMIN YANG ${ }^{1}$, JIANBING MA ${ }^{1}$, WENXING YU ${ }^{1}$ and XIUJIN CHEN ${ }^{1}$ \\ ${ }^{1}$ Department of Orthopedics, Hong-Hui Hospital, Xi'an Jiaotong University College of Medicine, Xi'an, Shaanxi 710054; \\ ${ }^{2}$ Department of Rheumatism, Immunity Branch, Xi'an No.5 Hospital, Xi'an, Shaanxi 710082, P.R. China
}

Received May 6, 2016; Accepted March 17, 2017

DOI: $10.3892 /$ etm.2018.5794

\begin{abstract}
Knee osteoarthritis is a degenerative disease that may develop due ageing, obesity, strain, congenital abnormal joints, joint deformity or trauma. It is caused by many factors, such as degradation of articular cartilage injury, joint edge and subchondral bone hyperplasia of reactivity. Platelet-rich plasma (PRP) is an autologous blood sample that contains highly concentrated platelets and multiple cell growth factors. PRP promotes synovial cell proliferation and differentiation and may recover cartilage morphology. In the present study, the clinical efficacy of PRP was investigated in patients with knee osteoarthritis aged between 18 and 30 years in a phase-III clinical study. Following an 8 -week baseline, patients with knee osteoarthritis were randomized into once-weekly, double-blind treatment with PRP (2-14 ml) or placebo groups. The results indicated that patients with osteoarthritis treated with PRP had modulated plasma concentrations of inflammatory factors and pro-angiogenic factors compared with the placebo group. Treatment responses were assessed by median percent reduction in inflammatory and pro-angiogenic factors and these improved with PRP treatment compared with the placebo. Clinical data indicated that PRP alleviated knee osteoarthritis and reduced humoral and cellular immune responses that led to beneficial effects on histological parameters. Inflammation was significantly alleviated in patients receiving PRP compared with the placebo group. The most common treatment-emergent adverse events in the presence of PRP were hypertension and proteinuria. In conclusion, treatment with PRP for patients with knee osteoarthritis presented beneficial effects in alleviating joint inflammation, cartilage destruction and bone damage, and repairing joint tissue. These results suggested that PRP may be a potential therapeutic agent for knee osteoarthritis.
\end{abstract}

Correspondence to: Professor Tuanmin Yang, Department of Orthopedics, Hong-Hui Hospital, Xi'an Jiaotong University College of Medicine, 76 Nanguo Road, Xi'an, Shaanxi 710054, P.R. China E-mail: yangtuanmin136@163.com

Key words: platelet-rich plasma, knee osteoarthritis, inflammation, angiogenesis

\section{Introduction}

Osteoarthritis is a degenerative disease characterized by joint pain, tenderness, stiffness, joint swelling, restricted movement and joint deformities (1). In recent years, an increasing number of patients are being diagnosed with osteoarthritis, which has a notable impact on human health and quality of life $(2,3)$. The causes of osteoarthritis are complex, and the pathogenesis related to this disease is not well understood (4). Osteoarthritis is divided into primary and secondary osteoarthritis according to the presence of local and systemic risk factors (5). Osteoarthritis is frequently diagnosed as rheumatoid arthritis or ankylosing spondylitis in clinical differential diagnosis $(1,6)$. Previous studies have indicated that agents targeting rheumatoid arthritis are relatively ineffective at present $(7,8)$. Therefore, there is an urgent requirement for more efficient treatments for osteoarthritis with minimal side effects.

Platelet-rich plasma (PRP) is an autologous blood sample that contains highly concentrated platelets and multiple cell growth factors. PRP promotes synovial cell proliferation and differentiation and may recover cartilage morphology (9). PRP also possesses multifunctional outcomes for the treatment of osteoarthritis, including osteonecrosis of the femoral head, cartilage injury and rheumatoid arthritis (9). Previous research has suggested that PRP exhibits benefits for injurious articular cartilage repair through the removal of harmful inflammation factors in patients with joint diseases (5). It has previously been reported that PRP was beneficial for rheumatoid arthritis without side effects through inhibition of inflammatory factor levels in synovial fluid (10). In addition, treatment-emergent adverse effects of PRP were not systematically reported in clinical investigation (10). The therapeutic outcomes of PRP isolated from autologous peripheral blood mononuclear cells, including blood products rich in cytokines, growth factors and other bio-active molecules, has been reported to be an efficient and innovative treatment protocol (11). Furthermore, a study by Sadabad et al (12) investigated the efficiency of PRP vs. hyaluronic acid for the treatment of knee osteoarthritis. A study Khoshbin et al (13) evaluated the available Level I and Level II literature on PRP as a therapeutic intervention in the management of symptomatic knee osteoarthritis in a systematic review. These reports demonstrated that intravenous injection of PRP was able to repair tendons and damaged articular 
bone, and primarily contribute to inflammatory elimination, which may have an important role in the morphology, collagen microarchitecture and subsequent mechanical properties of the injected vein.

Previous reports have indicated that inflammatory cytokines have an essential role in the initiation and development of osteoarthritis, targeting the synovium in joint diseases $(14,15)$. A study by Battaglia et al (16) reported the efficacy of ultrasound-guided intra-articular injections of PRP vs. hyaluronic acid for hip osteoarthritis, which demonstrated that PRP was more effective at reducing inflammation and relieving pain. Furthermore, a study by Laudy et al (17) demonstrated that PRP injections in patients with knee osteoarthritis resulted in decreased pain, improved function and global assessment, and changes regarding joint imaging. A study by Meheux et al (18) suggested that PRP injection significantly improved validated patient-reported outcomes in patients with symptomatic knee osteoarthritis at 6 and 12 months post-injection and indicated similarities and differences in outcomes based on the PRP formulations used in the analyzed studies. These clinical reports suggest that PRP exhibits a potential efficacy in treatment of osteoarthritis.

In the present study, the efficacy and outcomes of PRP were evaluated in younger patients, aged between 18 and 30 years, with knee osteoarthritis. Inflammatory factors were analyzed following treatment with PRP or a placebo. Treatment-emergent adverse events in patients with knee osteoarthritis after PRP were also investigated in the present study. Therapeutic efficacy of PRP for knee osteoarthritis was evaluated by clinical arthritis scores. The present findings suggested that PRP has a therapeutic effect on knee osteoarthritis progression and highlighted its potential as an anti-inflammatory treatment agent for knee osteoarthritis.

\section{Materials and methods}

Ethics statement. The present phase-III study (XAJT00699978) was carried out in strict accordance with the recommendations in the Guide for Honghui Hospital of Xi'an Jiaotong University College of Medicine (Xi'an, China) between February 2009 and October 2014. Ethical approval was granted by the Defense Research Committee on the Ethics of Experiments (Honghui Hospital, Xi'an Jiaotong University College of Medicine, Xi'an, China). All patients were required to review trial protocols and amendments, and subsequently provided their informed consent.

Patients. A total of 366 patients with knee osteoarthritis, aged 18-30 years and with a Karnofsky performance status (19) $\geq 80 \%$ were enrolled between February 2009 and October 2014 in the present study. Patients were randomly divided into two groups and once-weekly, double-blind trials were conducted in Xi'an Jiangtong University College of Medicine. A detailed description of the inclusion/exclusion criteria, allocation method and other details can be found in previously published studies $(20,21)$. A total of $8 \mathrm{ml}$ blood was harvested from the cubital vein and centrifuged for $5 \mathrm{~min}$ at $1,500 \mathrm{x}$ g. Patients with knee osteoarthritis received PRP $(2,4,8,10,12$ or $14 \mathrm{ml})$ treatment through intralesional injections and a placebo was used as a control. All patients were hospitalized throughout the duration of the study.

Study design. The present double-blind study was carried out in three phases: Baseline stage, double-blind treatment phase (4-week dose-titration treatment) and 4-week post-treatment stage for patients who volunteered to complete the ongoing extension study. Patients were randomized to once-weekly, double-blind treatment with PRP $(2,4,8,10,12$ or $14 \mathrm{ml})$ or placebo (10 $\mathrm{ml}$ normal saline). The optimal dosage of PRP was determined to be $10 \mathrm{ml}$.

Enzyme-linked immunosorbent assay (ELISA). The plasma concentration levels of hepatocyte growth factor (HGF; ab100687), intercellular adhesion molecule 1 (ICAM-1; ab83760), osteopontin (OPN; ab91655), platelet-derived endothelial cell growth factor (PD-ECGF; ab193691), vascular endothelial growth factor (VEGF; ab119576), platelet-derived growth factor (PDGF; ab21234), insulin-like growth factor 1 (IGF-1; ab108873), transforming growth factor- $\beta$ (TGF- $\beta$; ab92486), interferon- $\gamma$ (IFN- $\gamma$; ab177743), interleukin (IL)-6 (ab46402), IL-17A (ab83688), tumor necrosis factor- $\alpha$ (TNF- $\alpha$; ab181421), IL-1 $\beta$ and receptor activator of nuclear factor $\kappa$ B ligand (RANKL; ab100749) in patients with knee osteoarthritis were analyzed using ELISA kits (Abcam, Cambridge, UK). All procedures were carried out according to the manufacturer's instructions.

Magnetic resonance imaging (MRI) scanning. MRI was performed for all subjects to assess the therapeutic effects of PRP for knee osteoarthritis. A 3.0-T MRI scanner (Hitachi, Ltd., Tokyo, Japan) was used to evaluate the damaged area, joint inflammation and synovial proliferation as a marker for disease status. All data were transferred to the post-processing workstation. The data for the knee was recorded and used to calculate the degree of the lesion. Clinical osteonecrosis of the femoral head scores were evaluated using a scale of $0-2$, as previously described (22). The degree of knee osteoarthritis in the joints was scored on a scale of 0-5, as previously described (23).

Efficacy and safety assessments. Efficacy assessments, including the median percent reduction scores and response rate, were analyzed in patients with knee osteoarthritis from baseline and during the 4 -week treatment period. The median percent reduction scores were measured using the Karnofsky score and the analysis was conducted according to previous clinical studies $(24,25)$. Furthermore, assessments of the most frequent treatment-emergent adverse events were evaluated in all randomized patients who received the study drug and had at least one post-dose safety assessment. Dose-response analysis was conducted after the last PRP injection (26).

Statistical analysis. All data were presented as the mean \pm standard error of the mean. Differences between mean values were assessed using the Student's t-test for unpaired data. Comparisons of data between multiple groups were performed with analysis of variance followed by the Student-Newman-Keuls test. Continuous variables were reported as the mean with a $95 \%$ confidence interval (CI). Treatment effect was presented as the median reduction in 
Table I. Characteristics of patients with knee osteoarthritis.

\begin{tabular}{lcc}
\hline Parameter & PRP group & Placebo group \\
\hline Total patients $(\%)$ & $310(84.5 \%)$ & $56(15.5 \%)$ \\
Sex (F/M) & $150 / 160$ & $20 / 36$ \\
Performance status & & \\
(Karnofsky) & & \\
100 & 121 & 13 \\
90 & 112 & 25 \\
80 & 77 & 18 \\
Prior treatment & & 20 \\
Surgery & 85 & 18 \\
Antibody therapy & 103 & 18 \\
Others & 122 & \\
\hline
\end{tabular}

PRP, platelet-rich plasma; F, Female; M, Male.

knee osteoarthritis over the treatment period. Non-parametric Hodges-Lehmann estimates of median drug treatment effects and 95\% CI were provided. Response rates and treatment-emergent adverse events were analyzed using the $\chi^{2}$ test by SPSS 20.0 (IBM Corp., Armonk, NY, USA). P<0.05 was considered to indicate a statistically significant difference.

\section{Results}

Patient characteristics. A total of 366 patients with knee osteoarthritis were recruited and included in the present analysis. The mean age of patients was 24 years. All patients received either the agent (PRP) or placebo. Patients were randomized into two groups and treated with PRP or placebo. At baseline, mean age, body mass index and time since knee osteoarthritis diagnosis were similar between the two groups. The characteristics of patients with knee osteoarthritis are summarized in Table I. Notably, there was a higher percentage of male patients than female patients with knee osteoarthritis. Overall, 350 patients with knee osteoarthritis completed the maintenance period of the phase III studies.

Duration of treatment, dose-limiting toxicity and maximum tolerated dose (MTD). The median overall duration of PRP treatment was 8 weeks. Patients in the PRP group were treated with 2, 4, 8, 12 or $14 \mathrm{ml}$ of PRP. As shown in Table II, $12 \mathrm{ml}$ PRP once a week was identified as the MTD. Patients who received at least one dose of study therapy with post-baseline safety evaluation were included in the safety population. Following the last dose of PRP, it was observed that the common treatment-emergent adverse events were hypertension, diarrhea, vomiting, lethargy, rash, proteinuria, fatigue, constipation, weight decrease, appetite decrease, epistaxis, hypertriglyceridemia and peripheral edema (Table II). The most frequent treatment-emergent adverse events with a Common Toxicity Criteria grade $\geq 3$ were hypertension and proteinuria ( $\geq 5 \%$ each; Table III). Accordingly, treatment of PRP also presented a dose-dependent effect, and the optimum therapeutic dose PRP was identified as $10 \mathrm{ml}$. The
Table II. Treatment-related adverse events.

\begin{tabular}{lcccc}
\hline & & PRP & PRP \\
Adverse event & $\begin{array}{c}\text { Total } \\
(\mathrm{n}=54)\end{array}$ & $\begin{array}{c}2-4 \mathrm{ml} \\
(\mathrm{n}=12)\end{array}$ & $\begin{array}{c}\text { P-10 ml } \\
(\mathrm{n}=24)\end{array}$ & $\begin{array}{c}12-14 \mathrm{ml} \\
(\mathrm{n}=18)\end{array}$ \\
\hline Hypertension & 11 & 2 & 5 & 4 \\
Diarrhea & 4 & 1 & 2 & 1 \\
Proteinuria & 10 & 2 & 5 & 3 \\
Vomiting & 1 & 0 & 1 & 0 \\
Lethargy & 3 & 0 & 1 & 2 \\
Rash & 7 & 2 & 4 & 1 \\
Fatigue & 3 & 0 & 2 & 1 \\
Constipation & 3 & 1 & 0 & 2 \\
Weight decreased & 2 & 0 & 1 & 1 \\
Decreased appetite & 1 & 0 & 0 & 1 \\
Epistaxis & 4 & 2 & 1 & 1 \\
Hypertriglyceridemia & 2 & 1 & 1 & 0 \\
Edema peripheral & 3 & 1 & 1 & 1 \\
\hline
\end{tabular}

Treatment-emergent adverse events were analyzed by $\chi^{2}$ test. PRP, platelet-rich plasma.

Table III. Treatment-related hypertension and proteinuria by Common Toxicity Criteria grade.

\begin{tabular}{lcccc}
\hline & Total & $\begin{array}{c}\text { PRP } \\
2-4 \mathrm{ml} \\
(\mathrm{n}=12)\end{array}$ & $\begin{array}{c}\text { PRP } \\
8-10 \mathrm{ml} \\
(\mathrm{n}=24)\end{array}$ & $\begin{array}{c}\text { PRP } \\
12-14 \mathrm{ml} \\
(\mathrm{n}=18)\end{array}$ \\
\hline Adverse event & $(\mathrm{n}=54)$ & 2 & 5 & 4 \\
Hypertension & 11 & 0 & 2 & 2 \\
Grade 1 & 4 & 1 & 2 & 1 \\
Grade 2 & 4 & 1 & 1 & 1 \\
Proteinuria & 3 & 2 & 5 & 3 \\
Grade 1 & 10 & 0 & 1 & 1 \\
Grade 2 & 2 & 0 & 2 & 1 \\
Grade 3 & 3 & 2 & 2 & 1 \\
\hline
\end{tabular}

Treatment-emergent adverse events were analyzed by $\chi^{2}$ test. PRP, platelet-rich plasma.

data for the $12(n=28)$ and $14 \mathrm{ml}(\mathrm{n}=18)$ doses demonstrated a high number of adverse events, so these doses were considered inadvisable and few patients were treated at these dose levels.

Pharmacokinetic analysis. In the presence of PRP, it was observed that the majority of patients with knee osteoarthritis kept steady-state plasma concentrations after a last dose compared with placebo (Fig. 1A). Also, the dose response rates were observed via changes in Karnofsky performance score. An improvement in clinical score was observed regardless of the presence or absence of PRP (Fig. 1B). In addition, plasma concentrations of PRP increased in a dose-dependent manner in patients receiving PRP treatment (Fig. 2A). In the 
A

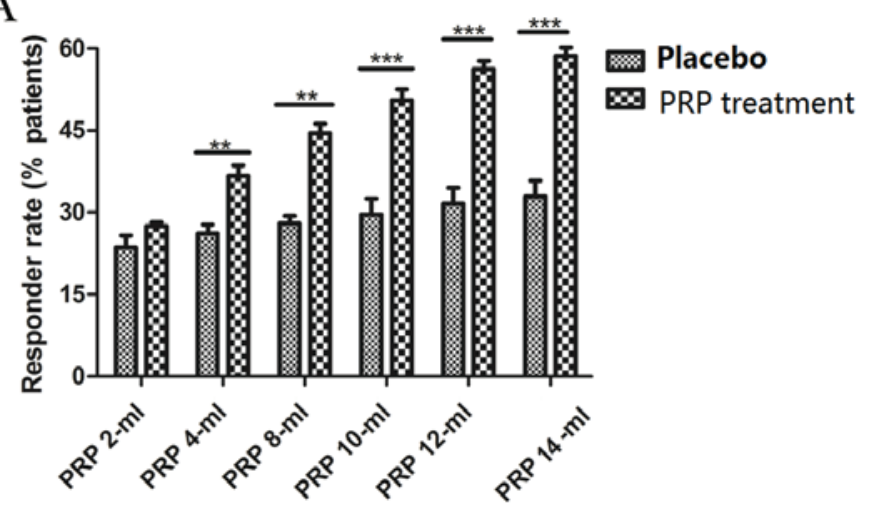

B

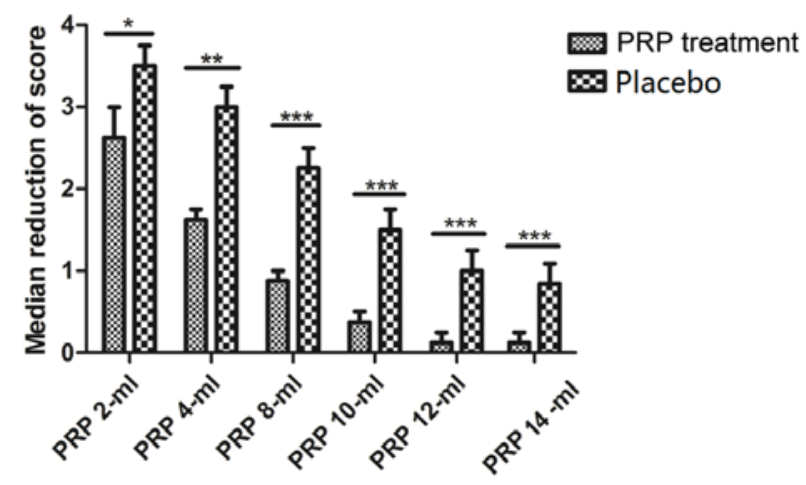

Figure 1. Response rate and median reduction of score for patients with knee osteoarthritis after treatment with PRP or placebo. (A) Response rate of patients with knee osteoarthritis during the treatment period. (B) Median reduction of score for patients with knee osteoarthritis 8 weeks from the baseline over double-blind period. Data are presented as the mean + standard error of the mean. ${ }^{*} \mathrm{P}<0.05,{ }^{* * *} \mathrm{P}<0.01$ and ${ }^{* * * *} \mathrm{P}<0.001$ vs. placebo. $\mathrm{PRP}$, platelet-rich plasma.
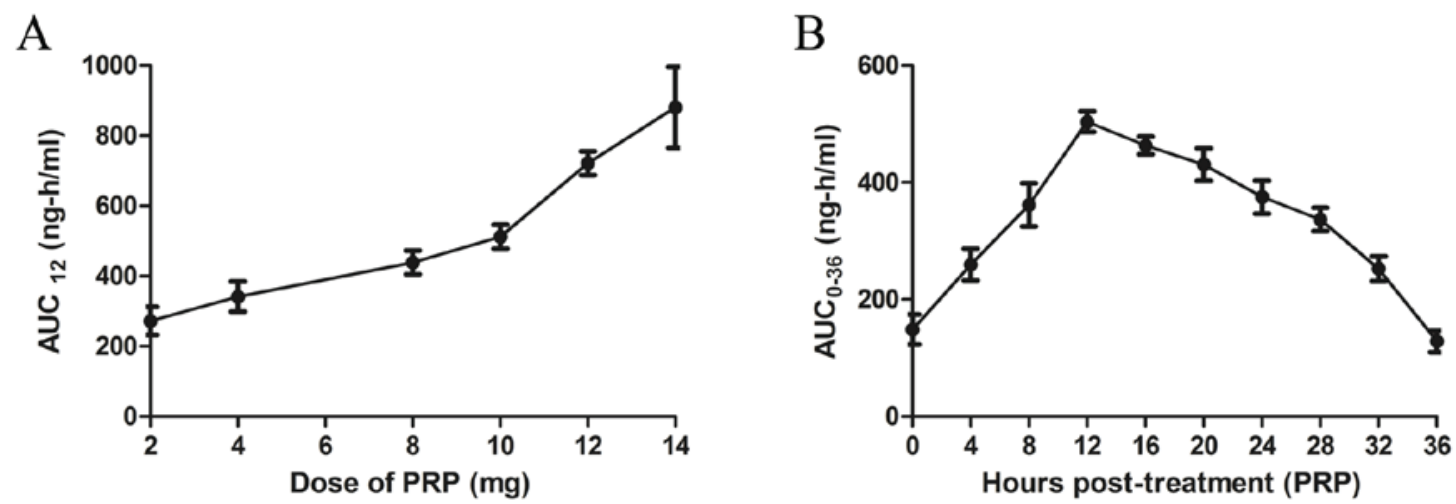

Figure 2. Pharmacokinetic analysis of PRP in patients with knee osteoarthritis. (A) Plasma concentrations of PRP with increasing dose. (B) Plasma concentrations of PRP $(10 \mathrm{ml})$ from $0-36 \mathrm{~h}$ post-treatment. Hodges-Lehmann estimates of median drug treatment effect and $95 \%$ confidence intervals are provided. Data are presented as the mean \pm standard error of the mean. AUC, area under the curve; PRP, platelet-rich plasma.

population pharmacokinetic analysis, PRP plasma concentration peaked at $12 \mathrm{~h}$ post-treatment (Fig. 2B).

Inflammatory factors. Plasma concentrations of inflammatory factors were analyzed in patients treated with PRP or placebo after the last dose of treatment. As demonstrated in Fig. 3A, plasma concentrations of IL-17A $(\mathrm{P}<0.001)$, IL-1 $\beta$ $(\mathrm{P}<0.01)$, TNF- $\alpha(\mathrm{P}<0.01)$, RANKL $(\mathrm{P}<0.01)$, IL-6 $(\mathrm{P}<0.01)$ and IFN- $\gamma(\mathrm{P}<0.001)$ were significantly downregulated after PRP treatment compared with the placebo treatment in an 8-week observation. As demonstrated in Fig. 3B, plasma concentrations of HGF $(\mathrm{P}<0.001)$, ICAM-1 $(\mathrm{P}<0.01)$, OPN $(\mathrm{P}<0.01)$, PD-ECGF $(\mathrm{P}<0.001)$, VEGF $(\mathrm{P}<0.001)$, PDGF $(\mathrm{P}<0.01)$, IGF-1 $(\mathrm{P}<0.001)$ and TGF- $\beta(\mathrm{P}<0.001)$ were significantly upregulated after PRP treatment compared with the placebo treatment.

Clinical arthritis scores. The response rates to PRP for patients with knee osteoarthritis were evaluated by clinical arthritis scores in the presence and absence of PRP. As demonstrated in Fig. 4A, PRP alleviated osteoarthritis and repaired damaged tissue determined by MRI parameters as compared with the placebo. As shown in Fig. 4B, PRP presented beneficial effects in preventing joint inflammation and synovial proliferation compared with the placebo.
Neovascularization and size of damaged area. It was observed that neovascularization was significantly promoted (Fig. 5A) and the damaged area significantly decreased (Fig. 5B) by PRP treatment compared with the placebo treatment (both $\mathrm{P}<0.001$ ). These clinical outcomes indicated that PRP at MTD $12 \mathrm{ml}$ dose improved the clinical features of knee osteoarthritis.

\section{Discussion}

The purpose of the present study was to demonstrate the efficacy and safety of PRP in patients with knee osteoarthritis, and in turn provide a rationale for PRP dosing recommendations. It was observed that PRP not only alleviated inflammation through humoral and cellular immune responses, but also had beneficial effects on MRI parameters., which was consistent with a previous study (20). As overall PRP therapeutic effects in the articular environment derive from an interaction with the pre-existing environment and other cells, and some surgical protocols involve the application of both platelets and cells, previous studies have investigated the effect of PRP on arthritis of different origins (25-27). Furthermore, the present investigation evaluated the MDT and treatment-emergent adverse events of PRP in the treatment of patients with knee osteoarthritis. 
A

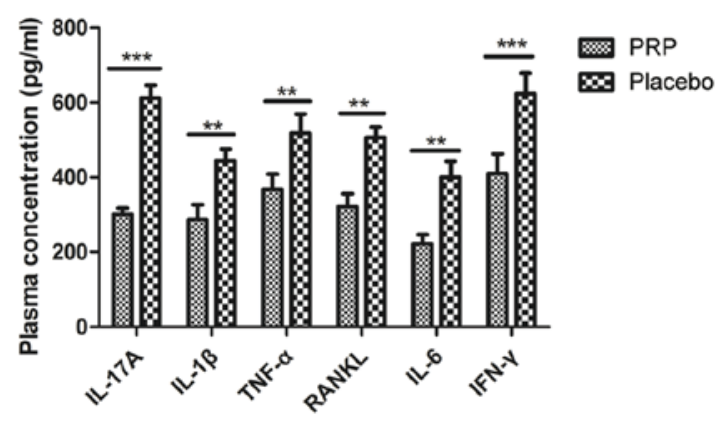

B

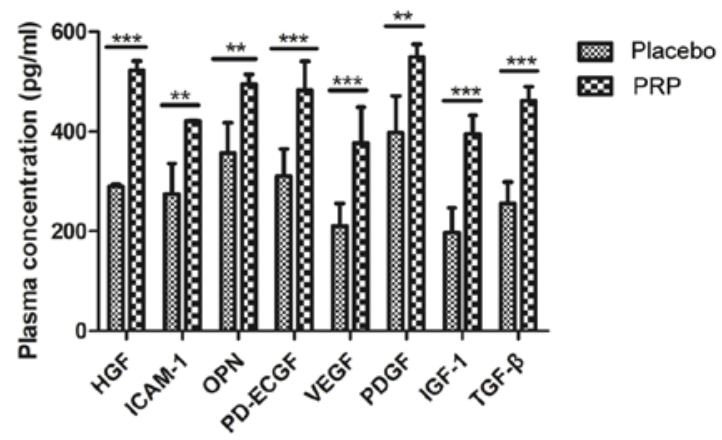

Figure 3. Inflammatory and pro-angiogenic factors in patients with knee osteoarthritis after treatment with PRP (10 ml) or placebo. (A) Plasma concentrations of IL-17A, IL-1 $\beta$, TNF- $\alpha$, RANKL, IL-6 and IFN- $\gamma$ after an 8-week observation. (B) Plasma concentration of HGF, ICAM-1, OPN, PD-ECGF, VEGF, PDGF, IGF-1 and TGF- $\beta$ after an 8 -week observation. Data are presented as the mean + standard error of the mean. ${ }^{* *} \mathrm{P}<0.01$ and ${ }^{* * *} \mathrm{P}<0.001$ vs. placebo. PRP, platelet-rich plasma; IL, interleukin; TNF- $\alpha$, tumor necrosis factor- $\alpha$; RANKL, receptor activator of nuclear factor $\kappa B$ ligand; IFN- $\gamma$, interferon- $\gamma$; HGF, hepatocyte growth factor; ICAM-1, intercellular adhesion molecule 1; OPN, osteopontin; PD-EGCF, platelet-derived endothelial cell growth factor; VEGF, vascular endothelial growth factor; PDGF, platelet-derived growth factor, IGF-1, insulin-like growth factor 1; TGF- $\beta$, transforming growth factor $\beta$.

A

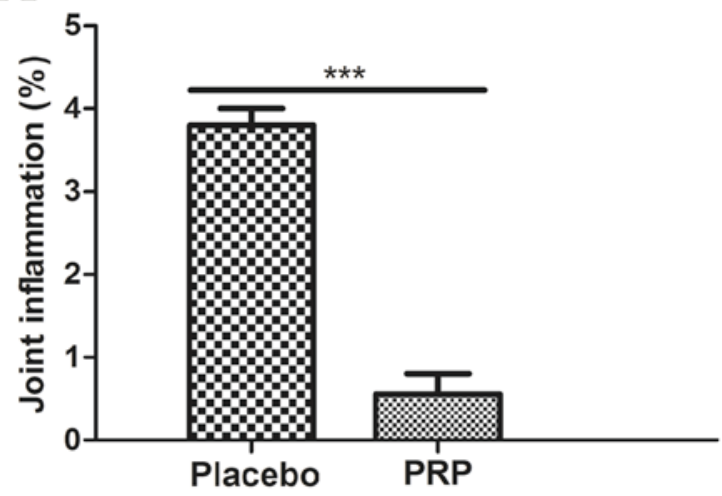

B

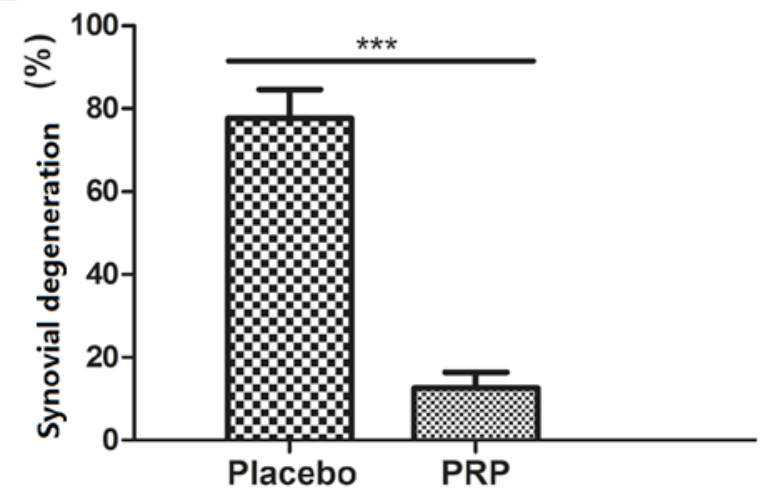

Figure 4. Analysis of joint inflammation and synovial proliferation in patients with knee osteoarthritis after treatment with PRP or placebo. (A) Effect of PRP treatment on joint inflammation in patients with knee osteoarthritis. (B) Effect of PRP treatment on synovial degeneration in patients with knee osteoarthritis. All patients underwent an 8-week maintenance period by injection of PRP $(10 \mathrm{ml})$. Data are presented as the mean + standard error of the mean. ${ }^{* * * *} \mathrm{P}<0.001$. PRP, platelet-rich plasma.

A

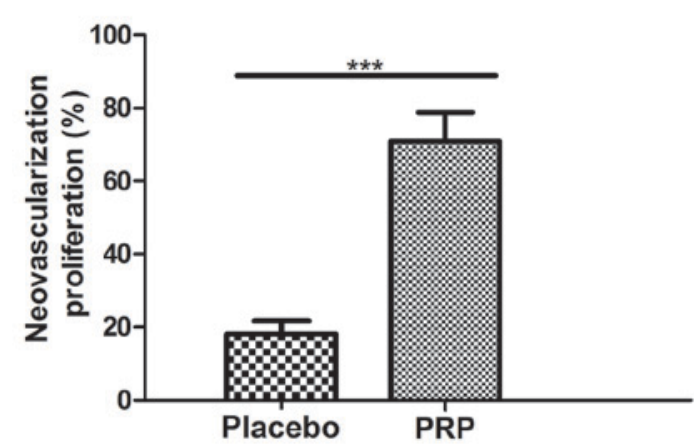

B

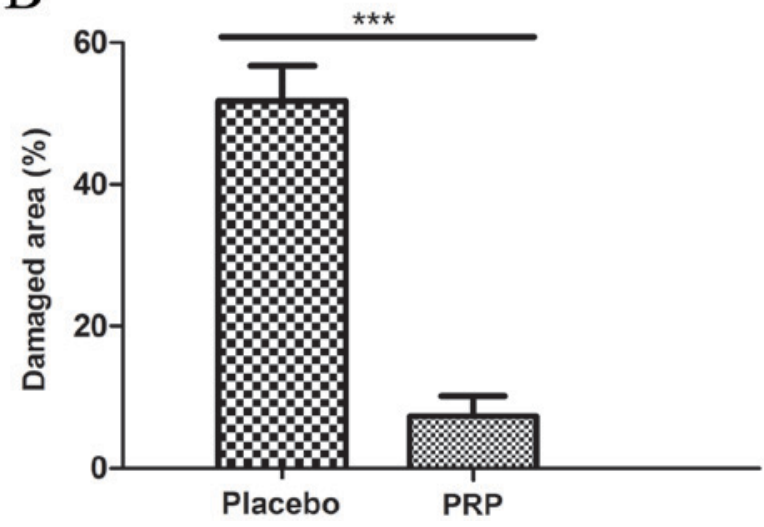

Figure 5. Analysis of neovascularization and the damaged area in synovial grafts in patients with knee osteoarthritis after treatment with PRP or placebo. (A) Destruction of the cartilage and neovascularization analysis after treatment with PRP or placebo. (B) Histological analysis of synovial damaged area after treatment with PRP or placebo. Data are presented as the mean + standard error of the mean. ${ }^{* * *} \mathrm{P}<0.001$. PRP, platelet-rich plasma.

The clinical data indicated that the most common treatment-emergent adverse events were hypertension and proteinuria $(\geq 10 \%)$, which was consistent with adverse events in the overall phase II study (27). The present results also indicated that PRP-treated patients with knee osteonecrosis had markedly improved synovial hyperplasia, inflammatory 
cell influx, destruction of the cartilage and angiogenesis analyzed by histological staining, as compared with the placebo-treated patients. These preclinical data suggest that PRP may be an effective agent for treatment of osteonecrosis of the femoral head.

Osteoarthritis is a serious chronic degenerative disease that affects patient health and quality of life (24). Although osteoarthritis may affect all human joints, knee osteoarthritis is the most common type among adolescents and adults (25). In recent years, non-surgical treatments for knee osteoarthritis have become more widely used, such as PRP, corticosteroid injection and hyaluronic acid $(25,28)$. A previous study described various methods for knee osteoarthritis treatment, including decompression with bone morphogenic proteins, growth factors, bone grafting and stem cells (29). In addition, a systematic review and meta-analysis compared the efficacy of PRP and hyaluronic acid for treatment of knee osteoarthritis and it was demonstrated that PRP injection was more effective than hyaluronic acid in a 2-year meta-analysis (12). These results were supported by the clinical outcomes of the present study in younger patients with knee osteoarthritis.

PRP is a prominent biomedical blood product that presents efficient outcomes for the treatment of patients with knee osteoarthritis, cartilage disorders and rheumatoid arthritis (30). As PRP has been approved as an agent for knee osteoarthritis therapy, it is important for clinicians to understand the potential pharmacokinetic interactions in order to maximize the therapeutic benefits of PRP and reduce the risk of treatment-emergent adverse events. The present study revealed that repeated administration of PRP (10 ml per week) relieved the pathogenesis of knee osteoarthritis. A previous report indicated that inflammatory cytokines form a complex regulatory signal network in osteonecrosis of the femoral head that is mediated by various intracellular kinase signaling pathways to regulate recruitment, stimulation and activation of autoimmune cells (31). Although the causes of knee osteoarthritis are not fully understood, laboratory and clinical evidence has suggested that inflammatory cytokines may contribute to its pathogenesis $(32,33)$. Theoretically, blocking inflammatory factor pathways may interrupt the inflammatory process and limit joint damage $(34,35)$. In the present study, clinical results indicated that inflammation factors were regulated following PRP treatment, which has not been previously reported. Furthermore, the stimulatory effects of PRP treatment have been demonstrated to promote proliferation and chondrogenic differentiation, which may produce beneficial molecules for the maintenance of articular cartilage perform $(23,36)$. The results of the present study suggest that PRP treatment improves inflammatory cell influx and angiogenesis.

Although a previous study has reported the direct effects of various drugs and PRP on knee osteoarthritis, it is essential to investigate the overall role of PRP in affecting the entire joint cytokine homeostasis (37). PRP has a long half-life and therefore is beneficial for treatment of knee osteoarthritis as PRP may be expected to degrade slowly (32). The results of the present study suggested that pharmacokinetic interactions of PRP are important determinants in optimizing therapy for knee osteoarthritis. Therefore, it is necessary for clinicians to monitor clinical responses and tolerability when patients are treated with PRP. In conclusion, the present findings indicate that PRP treatment for patients with knee osteoarthritis had beneficial effects in regulating inflammatory factors, and alleviating joint inflammation, cartilage destruction and bone damage.

\section{References}

1. Onuora S: Osteoarthritis: Molecular imaging detects activated macrophages. Nat Rev Rheumatol 12: 313, 2016.

2. Ma YW, Jiang DL, Zhang D, Wang XB and Yu XT: Radial extracorporeal shock wave therapy in a person with advanced osteonecrosis of the femoral head. Am J Phys Med Rehabil 95: e133-e139, 2016.

3. Lee GW, Park KS, Kim DY, Lee YM, Eshnazarov KE and Yoon TR: Results of total hip arthroplasty after core decompression with tantalum rod for osteonecrosis of the femoral head. Clin Orthop Surg 8: 38-44, 2016.

4. Roemer FW, Kwoh CK, Hannon MJ, Hunter DJ, Eckstein F, Grago J, Boudreau RM, Englund M and Guermazi A: Partial meniscectomy is associated with increased risk of incident radiographic osteoarthritis and worsening cartilage damage in the following year. Eur Radiol 27: 404-413, 2017.

5. Tang H, He S, Zhang X, Luo S, Zhang B, Duan X, Zhang Z, Wang W, Wang Y and Sun Y: A network pharmacology approach to uncover the pharmacological mechanism of XuanHuSuo powder on osteoarthritis. Evid Based Complement Alternat Med 2016: 3246946, 2016.

6. Poquet N, Williams Mand Bennell KL: Exercise for Osteoarthritis of the Hip. Phys Ther 96: 1689-1694, 2016.

7. Maricar N, Callaghan MJ, Parkes MJ, Felson DT and O'Neill TW: Clinical assessment of effusion in knee osteoarthritis-A systematic review. Semin Arthritis Rheum 45: 556-563, 2016.

8. Beumer L, Wong J, Warden SJ, Kemp JL, Foster P and Crossley KM: Effects of exercise and manual therapy on pain associated with hip osteoarthritis: A systematic review and meta-analysis. Br J Sports Med 50: 458-463, 2016.

9. Smyth NA, Haleem AM, Ross KA, Hannon CP, Murawski CD, Do HT and Kennedy JG: Platelet-rich plasma may improve osteochondral donor site healing in a rabbit model. Cartilage 7: 104-111, 2016.

10. Fu CJ, Sun JB, Bi ZG, Wang XM and Yang CL: Evaluation of platelet-rich plasma and fibrin matrix to assist in healing and repair of rotator cuff injuries: A systematic review and meta-analysis. Clin Rehabil 31: 158-172, 2017.

11. Vannini F, Di Matteo B and Filardo G: Platelet-rich plasma to treat ankle cartilage pathology - from translational potential to clinical evidence: A systematic review. J Exp Orthop 2: 2, 2015.

12. Sadabad HN, Behzadifar M, Arasteh F, Behzadifar M and Dehghan HR: Efficacy of platelet-rich plasma versus hyaluronic acid for treatment of knee osteoarthritis: A systematic review and meta-analysis. Electron Physician 8: 2115-2122, 2016.

13. Khoshbin A, Leroux T, Wasserstein D, Marks P, Theodoropoulos J, Ogilvie-Harris D, Gandhi R, Takhar K, Lum G and Chahal J: The efficacy of platelet-rich plasma in the treatment of symptomatic knee osteoarthritis: A systematic review with quantitative synthesis. Arthroscopy 29: 2037-2048, 2013.

14. Hodge JA, Kawabata TT, Krishnaswami S, Clark JD, Telliez JB, Dowty ME, Menon S, Lamba M and Zwillich S: The mechanism of action of tofacitinib - an oral Janus kinase inhibitor for the treatment of rheumatoid arthritis. Clin Exp Rheumatol 34: 318-328, 2016.

15. van der Goes MC, Jacobs JW and Bijlsma JW: Rediscovering the therapeutic use of glucocorticoids in rheumatoid arthritis. Curr Opin Rheumatol 28: 289-296, 2016.

16. Battaglia M, Guaraldi F, Vannini F, Rossi G, Timoncini A, Buda R and Giannini S: Efficacy of ultrasound-guided intra-articular injections of platelet-rich plasma versus hyaluronic acid for hip osteoarthritis. Orthopedics 36: e1501-e1508, 2013.

17. Laudy AB, Bakker EW, Rekers M and Moen MH: Efficacy of platelet-rich plasma injections in osteoarthritis of the knee: A systematic review and meta-analysis. Br J Sports Med 49: 657-672, 2015

18. Meheux CJ, McCulloch PC, Lintner DM, Varner KE and Harris JD: Efficacy of intra-articular platelet-rich plasma injections in knee osteoarthritis: A systematic review. Arthroscopy 32: 495-505, 2016.

19. Nahler G: Karnofsky performance status. Dictionary of Pharmaceutical Medicine: 101-102, 2009. 
20. Raeissadat SA, Rayegani SM, Hassanabadi H, Fathi M, Ghorbani E, Babaee M and Azma K: Knee osteoarthritis injection choices: Platelet- Rich Plasma (PRP) versus hyaluronic acid (A one-year randomized clinical trial). Clin Med Insights Arthritis Musculoskelet Disord 8: 1-8, 2015.

21. Rodriguez-Merchan EC: Intraarticular Injections of Platelet-rich Plasma (PRP) in the management of knee osteoarthritis. Arch Bone Jt Surg 1: 5-8, 2013.

22. Zalavras CG and Lieberman JR: Osteonecrosis of the femoral head: Evaluation and treatment. J Am Acad Orthop Surg 22: 455-464, 2014.

23. Bai F, Tian H, Niu Z, Liu M, Ren G, Yu Y, Sun T1, Li S and Li D: Chimeric anti-IL-17 full-length monoclonal antibody is a novel potential candidate for the treatment of rheumatoid arthritis. Int J Mol Med 33: 711-721, 2014.

24. Gobbi A, Lad D and Karnatzikos G: The effects of repeated intra-articular PRP injections on clinical outcomes of early osteoarthritis of the knee. Knee Surg Sports Traumatol Arthrosc 23: 2170-2177, 2015

25. Filardo G, Kon E, DI Matteo B, DI Marino A, Sessa A, Merli ML and Marcacci M: Leukocyte-poor PRP application for the treatment of knee osteoarthritis. Joints 1: 112-120, 2014.

26. Trotti A, Byhardt R, Stetz J, Gwede C, Corn B, Fu K, Gunderson L, McCormick B, Morrisintegral M, Rich T, et al: Common toxicity criteria: Version 2.0. an improved reference for grading the acute effects of cancer treatment: Impact on radiotherapy. Int J Radiat Oncol Biol Phys 47: 13-47, 2000.

27. Battaglia M, Guaraldi F, Vannini F, Buscio T, Buda R, Galletti S and Giannini S: Platelet-rich plasma (PRP) intra-articular ultrasound-guided injections as a possible treatment for hip osteoarthritis: A pilot study. Clin Exp Rheumatol 29: 754, 2011.

28. Jang SJ, Kim JD and Cha SS: Platelet-rich plasma (PRP) injections as an effective treatment for early osteoarthritis. Eur J Orthop Surg Traumatol 23: 573-580, 2013.

29. Pierce TP, Jauregui JJ, Elmallah RK, Lavernia CJ, Mont MA and Nace J: A current review of core decompression in the treatment of osteonecrosis of the femoral head. Curr Rev Musculoskelet Med 8: 228-232, 2015.
30. Kilincoglu V, Yeter A, Servet E, Kangal M and Yildirim M Short term results comparison of intraarticular platelet-rich plasma (prp) and hyaluronic acid (ha) applications in early stage of knee osteoarthritis. Int J Clin Exp Med 8: 18807-18812, 2015.

31. Lebouvier A, Poignard A, Cavet M, Amiaud J, Leotot J, Hernigou P, Rahmouni A, Bierling P, Layrolle P, Rouard H and Chevallier N: Development of a simple procedure for the treatment of femoral head osteonecrosis with intra-osseous injection of bone marrow mesenchymal stromal cells: Study of their biodistribution in the early time points after injection. Stem Cell Res Ther 6: 68, 2015.

32. Chiu WC, Lai YP and Chou MY: Humanization and characterization of an anti-human TNF- $\alpha$ murine monoclonal antibody. PLoS One 6: e16373, 2011.

33. Weisman MH: TNF and anti-TNF treatment in rheumatoid arthritis (RA). What we know and what we still need to know. Ryumachi 37: 142-143, 1997.

34. Elliot MJ, Maini RN, Feldmann M, Long-Fox A, Charles P, Katasikis P, Brennan FM, Bijl H, Ghrayeb J and Woody JN: Treatment of rheumatoid arthritis with chimeric monoclonal antibodies to tumor necrosis factor alpha. Arthritis Rheum 58 (2 Suppl): S92-S101, 2008.

35. Segal B, Rhodus NL and Patel K: Tumor necrosis factor (TNF) inhibitor therapy for rheumatoid arthritis. Oral Surg Oral Med Oral Pathol Oral Radiol Endod 106: 778-787, 2008.

36. Suzuki M, Tetsuka T, Yoshida S, Watanabe N, Kobayashi M, Matsui $\mathrm{N}$ and Okamoto T: The role of p38 mitogen-activated protein kinase in IL- 6 and IL-8 production from the TNF-alphaor IL-1beta-stimulated rheumatoid synovial fibroblasts. FEBS Lett 465: 23-27, 2000.

37. Görmeli G, Görmeli CA, Ataoglu B, Çolak C, Aslantürk O and Ertem K: Multiple PRP injections are more effective than single injections and hyaluronic acid in knees with early osteoarthritis: A randomized, double-blind, placebo-controlled trial. Knee Surg Sports Traumatol Arthrosc 25: 958-965, 2017. 